Ergod. Th. \& Dynam. Sys. (1989), 9, 587-604

Printed in Great Britain

\title{
The geometry of measured geodesic laminations and measured train tracks
}

\author{
HOWARD WEISS \\ Department of Mathematics, California Institute of Technology, \\ Pasadena, CA 91125, USA
}

(Received 12 December 1986 and revised 15 February 1988)

\begin{abstract}
Thurston and Kerckhoff have shown that the space of measured geodesic laminations on a hyperbolic Riemann surface serves as a non-linear model of the tangent space to Teichmüller space at the surface. In this paper we show that the natural map between these manifolds has stronger than Hölder continuous regularity.
\end{abstract}

\section{Introduction}

Thurston discovered that train tracks can be used to obtain a PL manifold structure on his space of measured geodesic laminations (MGLs) on a hyperbolic Riemann surface. Required are two fundamental constructions: the first is a method of constructing train track approximations of a MGL and the second is the construction of a MGL from a measured train track (MTT).

In this paper we study the geometry of the latter construction. We examine how the leaves of MGLs move about on a surface as one changes the weights on a train track, and in particular, we show that if two weights on a train track are $\varepsilon$ close, then the angle of leaves in the corresponding MGLs are $C \varepsilon \log ^{k}(1 / \varepsilon)$ close on the surface. Along the way, we study the growth rate of the number of train paths in a MTT with $n$ edges that correspond to (non empty) leaf packets in the MGL and show this function has polynomial growth. We also find a Train Path Uncertainty Principle which states if two weights on a MTT are $\varepsilon$ close, then the measures of any train path with $n$ edges can be no more than $n \varepsilon$ apart.

Kerchkoff has shown that the natural map between the space of MGLs on a Riemann surface and the tangent space of Teichmüller space at the surface is a homeomorphism. An immediate consequence of our main theorem is that this map, between the manifolds, has stronger than Hölder continuous regularity.

Since very little about MGLs and MTTs has appeared in print, we begin with several short sections containing background material, along with a sketch of Thurston's two fundamental constructions.

\section{Preliminaries on hyperbolic geometry}

Definition. A hyperbolic Riemann surface is a smooth oriented surface of genus $g \geq 2$ equipped with a metric of constant curvature -1 .

By the uniformization theorem, every hyperbolic Riemann surface $R$ is isometric to a surface of the form $H^{2} / \Gamma$ where $H^{2}$ is the hyperbolic plane and $\Gamma$ is a Fuchsian 
group (a discrete subgroup of $\operatorname{PSL}(2, R)$ ) acting by isometries, and isomorphic to $\pi_{1}(R)$. Let $\Sigma_{g}$ denote a fixed smooth, closed, oriented surface of genus $g$.

Definition. The Teichmüller space of genus $g, \mathscr{T}_{g}$, is the space of discrete and faithful representations from $\pi_{1}\left(\Sigma_{g}\right) \rightarrow \operatorname{PSL}(2, \mathbb{R})$ modulo conjugacy. Equivalently, $\mathscr{T}_{g}$ may be defined as the space of hyperbolic metrics on $\Sigma_{g}$ with two metrics identified if there is an isometry between them which is isotopic to the identity.

Every Mobius transformation $A$ acting on $H^{2}$ has a continuous extension to its boundary, denoted $S_{\infty}^{1}$, and called the 'circle at $\infty$ '. Pairs of distinct points on $S_{\infty}^{1}$ are in 1-1 correspondence with (oriented) geodesics in $\mathbb{H}^{2}$. Every hyperbolic Mobius transformation $A(|\operatorname{tr} A|>2)$ has exactly two fixed points both on $S_{\infty}^{1}$. The geodesic corresponding to the fixed points of $A \in \Gamma$ projects to the unique closed geodesic in $R$ contained in the free homotopy class of $i(A) \in \pi_{1}(\Sigma)$ under the isomorphism $i: \Gamma \rightarrow \pi(\Sigma)$.

Let $f$ be a homeomorphism between two Riemann surfaces $R_{1}$ and $R_{2}$, and let $f_{*}$ be the induced isomorphism between their uniformizing groups $\Gamma_{1}$ and $\Gamma_{2}$. This isomorphism gives a canonical 1-1 correspondence between elements of $\Gamma_{1}$ and $\Gamma_{2}$, and hence between closed geodesics on $R_{1}$ and $R_{2}$. Consequently, this isomorphism also induces a mapping between the sets of fixed points of elements of $\Gamma_{1}$ and $\Gamma_{2}$, which are dense in $S_{\infty}^{1}$.

The following theorem of Nielsen states that this mapping has a unique extension to a homeomorphism between the circles at infinity. This homeomorphism enables us to identify geodesics on the two surfaces.

THEOREM. (Nielsen, [T].) Let $f: R_{1} \rightarrow R_{2}$ be a homeomorphism between two closed hyperbolic Riemann surfaces. Then any lift of $f$ to a homeomorphism $\tilde{f}: \mathbb{H}^{2} \rightarrow \mathbb{H}^{2}$ has a unique continuous extension $\tilde{f}$ to a homeomorphism of $\mathrm{H}^{2} \cup S_{\infty}^{1}$, and the extension is invariant under isotopy, depending only on the lift and on $R_{1}$ and $R_{2}$. The extension is also equivariant so that the fixed points of any element $A_{1} \in \Gamma_{1}$ are taken to the fixed points of $A_{2} \in \Gamma_{2}$ where $\tilde{f}_{*}\left(A_{1}\right)=A_{2}\left(\tilde{f}_{*}: \Gamma_{1} \rightarrow \Gamma_{2}\right.$ induced by $\left.\tilde{f}\right)$.

\section{Measured geodesic laminations}

Let $R$ be a closed hyperbolic Riemann surface of genus $g$.

Definition. A geodesic lamination $\mathscr{G}$ on $R$ is a foliation of a closed subset of $R$ by complete, simple geodesics.

Let $\mathscr{A}$ denote the set of simple, smooth open arcs contained in $R$ which are transverse to $\mathscr{G}$, and have endpoint in $R-\mathscr{G}$.

Definition. A Measured geodesic lamination (MGL) is a pair $(\mathscr{G}, \mu)$, where $\mathscr{G}$ is a geodesic lamination and $\mu$ is a transverse measure satisfying:

(1) support $(\mu)=\mathscr{G}$

(2) If $t_{1}$ is homotopic to $t_{2}$ in $\mathscr{A}$, then $\mu\left(t_{1}\right)=\mu\left(t_{2}\right)$.

'Trivial' Example of a $M G L$. Let $(\mathscr{G}, \mu)$ be a finite disjoint union of simple closed geodesics $\phi_{1}, \ldots, \phi_{n}$ on $R$ with counting measure $\mu=\sum_{k=1}^{n} a_{k} \delta_{\phi_{k}}, a_{k}>0$. 
We will follow custom and denote the $\operatorname{MGL}(\mathscr{G}, \mu)$ by $\mu$. The following three theorems about MGL's are due to Thurston [T]:

THEOREM. If $\mu$ is a MGL on $R$ and $t \in \mathscr{A}$, then $t \cap \mu$ is either a discrete set or the union of a Cantor set and a discrete set. Moreover, the isolated points of $t \cap \mu$ are exactly the intersections of $t$ with simple closed leaves of $\mu$.

TheOREM. If $\mu$ is a MGL on $R$, then Hyperbolic Area $(\mu)=0$.

THEOREM. If $\mu$ is a MGL on $R$, then $R-\mu$ is a finite union of ideal polygons and Riemann surfaces with geodesic boundary.

The transverse measure on an MGL $\mu$ induces a Lebesgue-Stiltjes measure on arcs contained in $\mathscr{A}$. This allows us to integrate functions along transverse arcs. For a transverse arc $t \in \mathscr{A}$, let $\theta$ be the angle that the leaves of $\mu$ make with $t$ measured counterclockwise from $t$ to $\mu$. It is easy to see that $\theta$ is a Lipschitz function along $t$, and therefore may be integrated with respect to $\mu$. We define the total angle and total cosine of an arc $t \in \mathscr{A}$ by:

$$
\theta(t, \mu) \equiv \int_{t} \theta d \mu, \quad \cos (t, \mu) \equiv \int_{t} \cos \theta d \mu .
$$

Similarly, we define the total angle and total cosine of a simple closed geodesic $\phi$ by:

$$
\theta(t, \phi) \equiv \int_{\phi} \theta d \mu, \quad \cos (\phi, \mu) \equiv \int_{\phi} \cos \theta d \mu .
$$

Let $\operatorname{ML}(=\operatorname{ML}(R))$ denote the set of MGL's on $R$. Thurston defined the following topology on ML: given a finite set of $\operatorname{arcs}\left\{t_{k}\right\}_{k=1}^{n} \subset \mathscr{A}, \varepsilon>0$, and $\mu \in \mathrm{ML}$, a neighborhood basis of $\mu$ is given by:

$$
\begin{aligned}
\mathfrak{B}\left(\mu,\left\{t_{k}\right\}_{k=1}^{n}, \varepsilon\right) & =\left\{\nu \in \mathrm{ML}:\left|\left(\mu\left(t_{k}\right), \theta\left(t_{k}, \mu\right)\right)-\left(\nu\left(t_{k}\right), \theta\left(t_{k}, \nu\right)\right)\right|<\varepsilon,\right. \\
k & =1,2, \ldots, n\} .
\end{aligned}
$$

The following theorem of Thurston may be thought of as a strong existence statement for MGL's:

THEOREM. (Thurston, [T].) ML is homeomorphic to $\mathbb{R}^{6 \mathrm{~g}-6}$.

Let $\mathscr{S}$ denote the set of simple closed geodisics on $R$. We can embed $\mathscr{S} \times \mathbb{R}^{+}$in ML by sending $(\phi, r)$ to the MGL consisting of $\phi$ with $\mu=r \cdot \delta_{\phi}$.

TheOREM. (Thurston, [T].) $\mathscr{S} \times \mathbb{R}^{+}$is dense in $M L$.

This theorem allows one to extend notions defined for simple closed geodesics such as length and Dehn twist to MGL's.

Given two Riemann surfaces $R_{1}$ and $R_{2}$ of genus $g$, along with an isomorphism between their uniformizing groups, we have seen that the mapping between their circles at infinity may be used to identify MGL's on the two Riemann surfaces.

THEOREM. (Thurston, [T].) Suppose $R_{1}$ amd $R_{2} \in \mathscr{T}_{g}$, thought of as hyperbolic structures on a fixed surface $\Sigma_{g}$. Let $\mu_{R_{1}}$ be a $M G L$ on $R_{1}$ and let $\mu_{R_{2}}$ be the 'corresponding' $M G L$ on $R_{2}$. Then the leaves of $\mu_{R_{1}}$ are isotopic to the leaves of $\mu_{R_{2}}$ on $\Sigma_{g}$. 


\section{Train tracks}

Train tracks serve as 'finite approximations' to MGL's and also allow us to define a PL (Piecewise linear) manifold structure on ML. The reader is referred to [HP] for an exhaustive treatment of train tracks.

A train track on $\Sigma_{g}$ is a branched 1-dimensional submanifold with several added technical conditions to assure compatibility with MGLs.

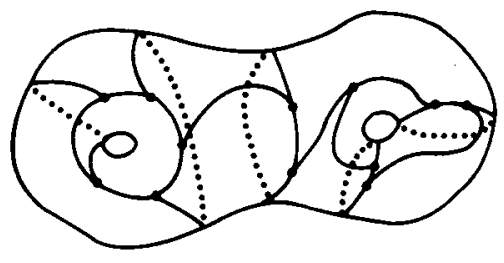

FIGURE 1. Train track.

Definition. A curve $c$ is carried by $\tau$ if there exists a $C^{1}$ map $\phi: \Sigma_{g} \rightarrow \Sigma_{g}$ such that $\phi(c) \subset \tau, \phi$ is homotopic to the identity, and $\left.d \phi\right|_{\text {tangent spaceto } c} \neq 0$.

Definition. An (infinite) train path is a map $\rho: \mathbb{R} \rightarrow \tau$ which is a $C^{1}$ immersion and $\rho(k, k+1)=$ edge and $\rho(k)=$ switch for all $k \in Z$.

Definition. A measure $\lambda$ for $\tau$ is an assignment of a non-negative number $\mu(e)$ to each edge $e \subset \tau$ satisfying at each switch $V$ the 'switch condition':

$$
\mu\left(e_{1}\right)+\mu\left(e_{2}\right)+\cdots+\mu\left(e_{s}\right)=\mu\left(e_{s+1}\right)+\cdots+\mu\left(e_{t}\right)
$$

where $e_{1}, \ldots, e_{s}$ are the incoming edges at $v$ and $e_{s+1}, \ldots, e_{t}$ are the outgoing ones.

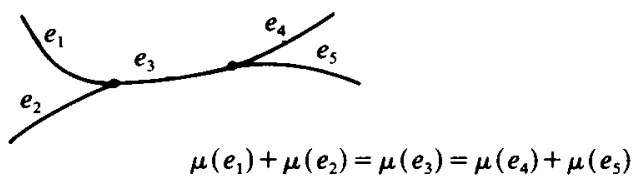

FIGURE 2. Switch condition.

The set of measures on $\tau$ is a convex Euclidean cell and is denoted $V(\tau)$.

Definition. $\tau$ is recurrent if for each edge $e \subset \tau$, there exists non-trivial closed curves $C_{e}$ carried by $\tau$ with supporting map $\phi$ and $\phi\left(C_{e}\right) \supset e$, or equivalently, $\tau$ supports a measure with all edge weights positive.

Definition. A simple closed curve $c$ hits $\tau$ efficiently if there does not exist a (not necessarily embedded) bigon $B \subset \Sigma=\tau-c$ with $\partial B$ a union of two $C^{1}$ segments $S_{1} \subset C$ and $S_{2} \subset \tau$.

Definition. $\tau$ is transversely recurrent if for each edge $e \subset \tau$, there exists a non-trivial simple closed curve $C_{e}$ hitting $\tau$ efficiently and $C_{e} \cap e \neq \phi$.

Definition. $\tau$ is birecurrent if it is both recurrent and transversely recurrent.

We will assume that all edges are open and all switches are trivalent. 


\section{Fundamental construction \# 1}

We will now outline two fundamental constructions of Thurston: The first will be a method of constructing train track approximations of an MGL and the latter will be the construction of an MGL from a transversely recurrent train track.

Construction \#1. E-train track approximation of an $M G L \mu$. Before beginning the construction, we need the following preliminary result for a Riemann surface $R$ with finite area.

Definition. $\boldsymbol{R}_{(0, \varepsilon]}=\{p \in R$ : there exists non-trivial closed loop through $p$ with length $\leq \varepsilon\}$.

Proposition. (Margulis [BE].) There exists a universal constant $C$ such that $R_{(0, \varepsilon]}$, $\varepsilon \leq C$ is the disjoint union of cusps and tubular neighborhoods of simple closed geodesics with length $\leq \varepsilon$.

Step 1. Since $R-\mu=R_{1} \cup R_{2} \cup \cdots \cup R_{N}, N<\infty$, where each $R_{k}$ is either an ideal polygon (generically a triangle) or a Riemann surface with geodesic boundary, the double of $R_{k}, d R_{k}$, is a Riemann surface with finite area. Consider $\left(d R_{k}\right)_{(0,2 \varepsilon]}$. $\left(d R_{k}\right)_{(0,2 \varepsilon]}$ has a canonical foliation by curves of constant curvature, i.e., equidistant curves about a short geodesic and horocycles about cusps.

Step 2. Transfer the partial foliations on $d R_{k}$ to $R_{k}$ and then back to $R$.

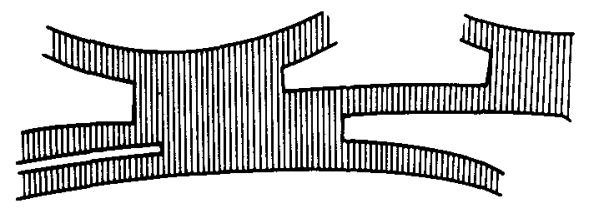

FIgURE 3. Partial foliation on $R$.

LEMMA. (Thurston, $\left[\mathbf{W}_{1}\right]$.) The partial foliations on the $R_{k}$ transferred to $R$ extend to a unique Lipschitz foliation $\mathscr{F}$ on the closure of $\bigcup_{k=1}^{N}\left(R_{k}\right)_{(0, \varepsilon]}$.

Step 3. Now collapse each leaf of $\mathscr{F}$ down to a point. A well-known theorem of Moore [MOO] insures that the quotient 'object' $\tilde{R}$ is a (topological) surface. The image of $\mathscr{F}$ under the quotient map is the $\varepsilon$-train track approximation to $\mu$ denoted $\tau_{\varepsilon}$.

Remark. It is clear that the switches of $\tau_{\varepsilon}$ come from collapsing leaves of $\mathscr{F}$ near configurations such as:

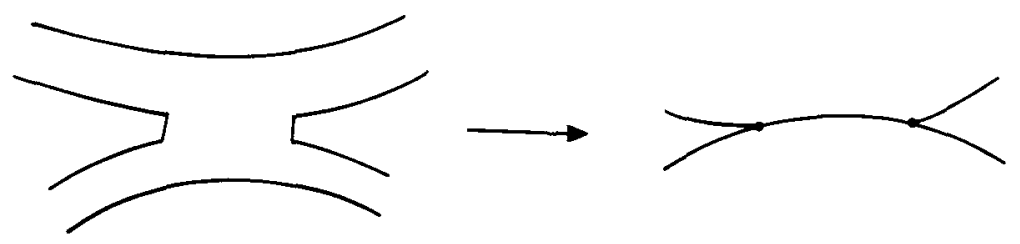

FIGURE 4 
Since the number of switches cannot be greater than the total number of cusps in the $R_{k}$, it follows from simple Euler characteristic considerations that there are at most $4 g-4$ complimentary regions $R_{k}$ and \# switches of $\tau_{\varepsilon} \leq 3(4 g-4)=12 g-12$.

Step 4. (optional) (Thurston, [T, MOR].) Given $\delta>0$, there exists $\varepsilon_{0}>0$ such that if $\varepsilon<\varepsilon_{0}, \tau_{\varepsilon}$ (living on the quotient surface $\tilde{R}$ ) can be pulled back to a train track on $R$ with geodesic curvature $<\delta$.

Our train track approximation $\tau_{\varepsilon}$ comes equipped with a natural measure. Each branch is assigned the weight corresponding to the total mass of the leaves in $\mu$ which collapse onto it. It is clear this defines a measure on $\tau_{\varepsilon}$.

It is important to keep in mind that train tracks are topological objects having finite combinatorics. Thurston advocates not studying MGL's on a microscopic level, i.e., thinking about individual leaves, but to study them on a macroscopic level using $\varepsilon$-train track approximation.

\section{Fundamental construction \#2}

Construction \#2. Construction of an MGL from a measured transversely recurrent train track.

Let us start with a transversely recurrent train track $\tau$ on $R$ with measure $\lambda$.

Step. 1. [Construction of a Euclidean model for $(\lambda, \lambda)$.] For each edge $e \subset \tau$ take a Euclidean strip of width $k \cdot \lambda(e)$, where $k$ is a scaling factor, $k>0$, and splice these strips together obeying the switch condition at each vertex. The resulting object has a natural horizontal (singular) foliation obtained from the natural foliation on the Euclidean strips.

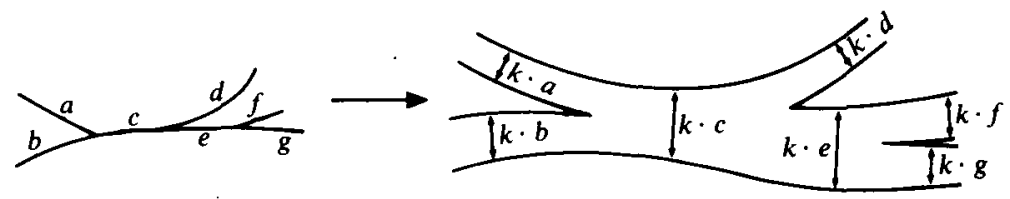

FIGURE 5. Euclidean model.

Step 2. Choose $k$ small enough such that the foliated model $\subset \mathbb{R}^{3}$ embeds into $R$ with $\tau$ as a spine. The model is not, and will not be isometrically embedded. All singular leaves of the foliation should be regarded as a pair of leaves, an 'upper' and a 'lower', making a consistent choice at each switch.

Step 3. Lift $\mathscr{L}$ to a partial foliation $\tilde{\mathscr{L}}$ in $\mathrm{H}^{2}$. The transverse recurrence of $\tau$, insures that each $\tilde{l} \in \tilde{\mathscr{L}}$ has well defined endpoints on $S_{\infty}^{1}$ [HP]. Now replace each leaf $\tilde{l} \in \tilde{\mathscr{L}}$ with the geodesic having the same endpoints on $S_{\infty}^{1}$. Denote the new collection of geodesic leaves $\tilde{\mathscr{G}}$.

\section{Remarks.}

(1) Many leaves of $\tilde{\mathscr{L}}$ may amalgamate to create one leaf in $\tilde{\mathscr{G}}$.

(2) The assumption of transverse recurrence on $\tau$ is only needed to insure that the leaves of $\tilde{\mathscr{F}}$ have well defined endpoints on $S_{\infty}^{1}$. If it is known that $\tau$ has geodesic 
curvature strictly less than one, then it follows from elementary hyperbolic geometry that the lifts have well defined endpoints on $S_{\infty}^{1}$.

$\tilde{G}$ is a geodesic lamination on $\mathbb{H}^{2}$, i.e., $\tilde{G}$ is a closed disjoint union of simple geodesics. Now project $\tilde{\mathscr{G}}$ down to a geodesic lamination $\mathscr{G}$ on $\mathbb{R}$.

Step 4. [Construction of the transverse measure on $\mathscr{G}$.] We now construct a measure on arcs in $\mathscr{A}$ (open smooth arcs contained in $R$ which are transverse to $\mathscr{G}$ ). Let $\mathscr{A}^{*} \subset \mathscr{A}$ consists of those arcs $t \in \mathscr{A}$ such that $t$ intersects precisely those leaves of $\mathscr{G}$ which collapse onto some edge $e_{t} \subset \tau$.

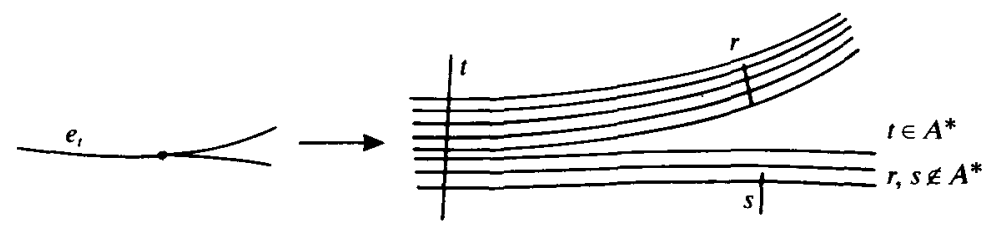

Figure 6

For $t \in \mathscr{A}^{*}$ define $\mu(t)=\lambda\left(e_{t}\right)$.

Claim. The transverse measure $\mu$ on $\mathscr{A}$ is completely determined by $\mu$ on $\mathscr{A}^{*}$.

$$
\mu(t)=\mu\left(t_{1}^{1}\right)+\mu\left(t_{1}^{2}\right)=\mu\left(t_{2}^{1}\right)+\mu\left(t_{2}^{2}\right)+\mu\left(t_{2}^{3}\right)=\lambda\left(e_{t_{2}}\right)+\lambda\left(e_{t_{2}^{2}}^{2}\right)+\lambda\left(e_{t_{2}^{3}}^{3}\right) .
$$

Since the leaves of $\mathscr{G}$ are geodesics, they diverge from each other 'exponentially fast', hence the endpoints of arcs $t \in \mathscr{A}$ are only 'finitely buried' in $\mathscr{G}$. The mass of an arc $t \in \mathscr{A}$ is computed by 'dragging' the arc along $\mathscr{G}$ until it splits into a union of arcs all of which are contained in $\mathscr{A}^{*}$.

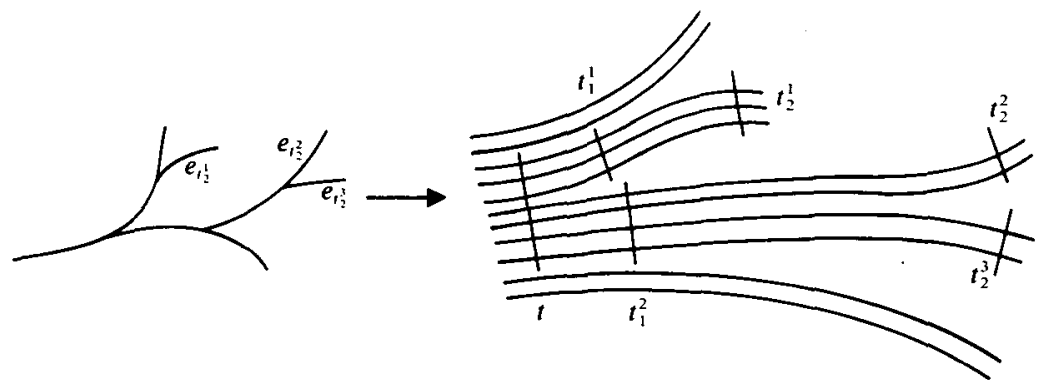

FIGURE 7. 'Dragging' a transverse arc.

THEOREM. (Thurston, [HP].) If $\tau$ is a birecurrent train track and $\lambda_{1}, \lambda_{2} \in V(\tau)$, then the MGL's $\mu_{1}$ and $\mu_{2}$ constructed from $\left(\tau, \lambda_{1}\right)$ and $\left(\tau, \lambda_{2}\right)$ coincide iff $\lambda_{1}=\lambda_{2}$.

6. Measured train tracks and train track coordinates on $M L$

There is a natural equivalence relation on measured train tracks such that if $\left(\tau_{1}, \lambda_{1}\right)$ and $\left(\tau_{2}, \lambda_{2}\right)$ are two equivalent transversely recurrent train tracks, then the MGL's constructed from them coincide. 
Definition. $M T$ is the space of equivalence classes of all measured $\operatorname{tr}^{\mathrm{i}}$ equipped with the quotient topology

$$
\bigsqcup_{\tau \in R} V(\tau) \stackrel{\pi}{\rightarrow} M T .
$$

The following three theorems of Thurston are proved in [HP].

THEOREM A. MT is homeomorphic to $\mathbb{R}^{6 g-6}$.

THEOREM B. Let $\psi$ denote the mapping which constructs the MGL from a t recurrent measured train track.

(a) $\psi$ is a continuous injection and $\pi$ is a continuous mapping.

(b) $\eta$ is a bijection.

(c) Since $M T, M L$ are homeomorphic to $\mathbb{R}^{6 \mathrm{~g}-6}$, it follows

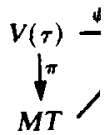
from 'invariance of domain' that $\eta$ is a homeomorphism.

Theorem C. $M L$ is a PL (Piecewise Linear) manifold with PL coordinat $\psi_{\tau}: V(\tau) \rightarrow M L$, where $\tau$ is a transversely recurrent maximal (there does proper subtrack) train track.

Let $M L$ denote the $P L$ manifold structure on $M L$ given by train track manifold structure on $M L$ may alternately be described as follows: Let $M L$ be a local parametrization for $M L$, and let $e_{1}, \ldots, e_{N}$ be the edges of $N$ arcs $\alpha_{1}, \ldots, \alpha_{N}$ transverse to all leaves in Im $\left(\psi_{\tau}\right)$ such that $\alpha_{k}$ intersec those leaves in $\operatorname{Im}\left(\psi_{\tau}\right)$ which collapse onto $e_{k}, k=1,2, \ldots, N$. Coordi are given by $\left(\operatorname{Im}\left(\psi_{\tau}\right), f_{\tau}\right)$, where:

$$
\begin{gathered}
f_{\tau}: \operatorname{Im}\left(\psi_{\tau}\right) \subset M L \rightarrow \mathbb{R}^{N} \\
\mu \rightarrow\left(i\left(\mu, \alpha_{1}\right), \ldots, i\left(\mu, \alpha_{N}\right)\right) .
\end{gathered}
$$

We see that train track coordinates are equivalent to intersection numb 'strategically placed' transverse arcs.

\section{Packet uncertainty principle}

Let $\tau$ be a train track on $R$.

Definition. A finite train path in $\tau$ is a mapping $\rho:[0, N] \rightarrow \tau, N \in \mathbb{Z}^{+}$ $C^{1}$-immersion, $\left.\rho(k, k+1)\right)=$ edge and $\rho(k)=$ switch for $0 \leq k \leq N$.

We will usually denote a finite train path by listing its edges.

A measure $\lambda$ on $\tau$ induces a measure on finite train paths in $\tau$. The $\mathrm{F}$ inductive, and is illustrated below:

Let $(\tau, \lambda)$ be a MTT and let $\left\{e_{1}, e_{2}, \ldots, e_{n}\right\}$ be a finite train path wi We first define a subinterval of $\left[0, \lambda\left(e_{n}\right)\right], I\left\{e_{1}, \ldots, e_{n}\right\}$, by inductio 1 define $\lambda\left\{e_{1}, \ldots, e_{n}\right\}=$ length $\left(I\left\{e_{1}, \ldots, e_{n}\right\}\right)$. If $n=1, I\left\{e_{1}\right\}=\left[0, \lambda\left(e_{1}\right.\right.$ $I\left\{e_{1}, \ldots, e_{n-1}\right\}$ has been defined. There are four possible configu $\left\{e_{1}, \ldots e_{n}\right\}$ : 

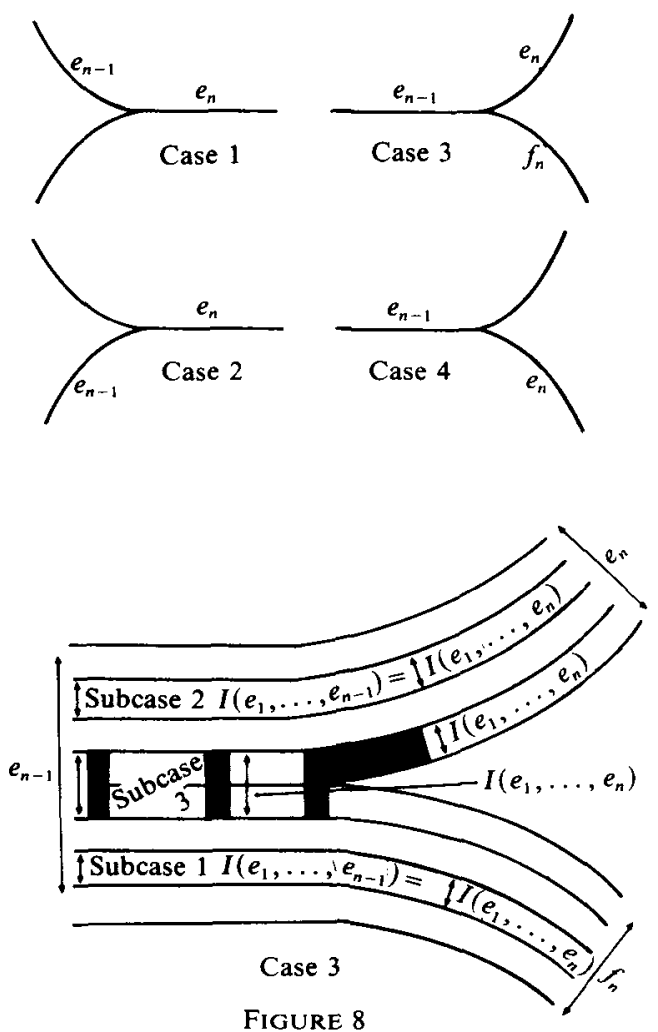

Cases 1 and 2 are trivial. $I\left\{e_{1}, \ldots, e_{n}\right\}=I\left\{e_{1}, \ldots, e_{n-1}\right\}$, hence $\lambda\left\{e_{1}, \ldots, e_{n}\right\}=$ $\lambda\left\{e_{1}, \ldots, e_{n-1}\right\}$.

Case 3 has 3 subcases:

subcase 1: $\max I\left\{e_{1}, \ldots, e_{n-1}\right\} \leq f_{n}$

$$
I\left\{e_{1}, \ldots, e_{n}\right\}=I\left\{e_{1}, \ldots, e_{n}\right\}
$$

hence $\lambda\left\{e_{1}, \ldots, e_{n}\right\}=\lambda\left\{e_{1}, \ldots, e_{n-1}\right\}$

subcase $2: \min I\left\{e_{1}, \ldots, e_{n-1}\right\} \geq f_{n}$

$$
I\left\{e_{1}, \ldots, e_{n}\right\}=\left[\min I\left\{e_{1}, \ldots, e_{n-1}\right\}-f_{n}, \max I\left\{e_{1}, \ldots, e_{n-1}\right\}-f_{n}\right],
$$

hence $\lambda\left\{e_{1}, \ldots, e_{n}\right\}=\lambda\left\{e_{1}, \ldots, e_{n-1}\right\}$

subcase 3: $\min I\left\{e_{1}, \ldots, e_{n-1}\right\}<f_{n}$ and $\max I\left\{e_{1}, \ldots, e_{n-1}\right\}>f_{n}$

$$
I\left\{e_{1}, \ldots, e_{n}\right\}=\left[0, \max I\left\{e_{1}, \ldots, e_{n-1}\right\}-f_{n}\right],
$$

hence $\lambda\left\{e_{1}, \ldots, e_{n}\right\}=\max I\left\{e_{1}, \ldots, e_{n-1}\right\}-f_{n}$

Case 4 is identical to Case 3 and is omitted.

Definition. Given two measures $\lambda_{1}$ and $\lambda_{2}$ on $\tau$,

$$
\left|\lambda_{1}-\lambda_{2}\right|_{\infty}=\max _{e \subset \tau}\left|\lambda_{1}(e)-\lambda_{2}(e)\right|,
$$

where the maximum is over all edges $e \subset \tau$. 
Example.

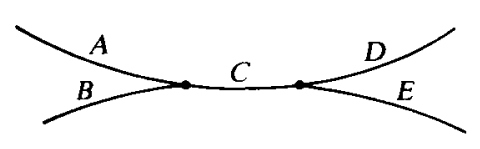

\begin{tabular}{c|c|cc}
\multicolumn{2}{r|}{$\lambda_{1}$} & $\lambda_{2}$ & \\
\hline$A$ & $a$ & $a+\delta$ & \\
$B$ & $b$ & $b-\delta$ & \\
$C$ & $c$ & $c$ & $a \geq d$ \\
$D$ & $d$ & $d-\delta$ & \\
$E$ & $e$ & $e+\delta$ & $\delta>0$ small
\end{tabular}

FIGURE 9

It is clear that $\left|\lambda_{1}-\lambda_{2}\right|_{\infty}=\delta$. Consider the train path $P=\{A C E\}$.

$$
\begin{aligned}
& \lambda_{1}(P)=a-d \\
& \lambda_{2}(P)=(a+\delta)-(d-\delta)=a-d+2 \delta .
\end{aligned}
$$

Hence, $\left|\lambda_{1}(P)-\lambda_{2}(P)\right|=2 \delta=2\left|\lambda_{1}-\lambda_{2}\right|_{\infty}$. This trivial example illustrates the phenomenon of 'train path uncertainty'.

Lemma 1.1. [Train Path Uncertainty.] Let $\tau$ be a train track on $R, \lambda_{1}, \lambda_{2} \in V(\tau)$, and let $P$ be a train path with $n$ edges. Then

$$
\left|\lambda_{1}(P)-\lambda_{2}(P)\right| \leq n\left|\lambda_{1}-\lambda_{2}\right|_{\infty} .
$$

Proof. Let $P=\left\{e_{1}, \ldots, e_{n}\right\}$ be a train path with $n$ edges. There are at most $n$ edges of $\tau$ incident upon $P$. Let $f_{1}, \ldots, f_{n}$ denote those edges of $\tau$ that are incident to $P$, where $e_{k}$ and $f_{j}$ are incident upon the same switch. It follows from the previous discussion on weights on finite train paths, that

$$
\lambda_{1}(P)=\lambda_{1}\left(e_{1}\right) \pm \lambda_{1}\left(f_{2}\right) \pm \lambda_{1}\left(f_{3}\right) \pm \cdots \pm \lambda_{1}\left(f_{n}\right) .
$$

Then,

$$
\lambda_{2}(P)=\left[\lambda_{1}\left(e_{1}\right) \pm \delta_{1}\right] \pm\left[\lambda_{1}\left(f_{2}\right) \pm \delta_{2}\right] \pm \cdots \pm\left[\lambda_{1}\left(f_{n}\right) \pm \delta_{n}\right]
$$

where the pattern of + or - between the bracketed terms in $\lambda_{2}(P)$ is the same as in $\lambda_{1}(P)$, and $0 \leq \delta_{1}, \delta_{2}, \ldots, \delta_{n} \leq\left|\lambda_{1}-\lambda_{2}\right|_{\infty}$. There is no bound on the sign combinatorics, and the worst case of the sign pattern could occur, hence $\left|\lambda_{1}(P)-\lambda_{2}(P)\right| \leq$ $n\left|\lambda_{1}-\lambda_{2}\right|_{\infty}$.

We will now translate the uncertainty principle into MGL language. Given an MGL $\mu$ on $R$, let $(\tau, \lambda)$ be a train track approximation to $\mu$.

Definition. Let $P=\left\{e_{1}, \ldots, e_{n}\right\}$ be a train path in $\tau$. The leaf packet in $\mu$ corresponding to $P, \mathscr{P}$, consists of all those segments of leaves in $\mu$ which collapse exactly onto $P$.

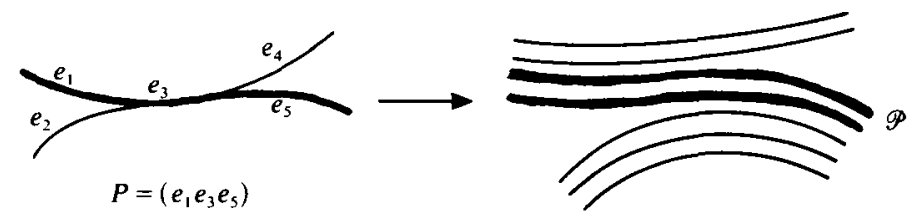

Figure 10

Note the leaf packet corresponding to $P=\left\{e_{2} e_{3} e_{4}\right\}$ is empty. 
COROLlary 1.2 [Leaf Packet Uncertainty Principle.] Let $\tau$ be a birecurrent train track on $R, \lambda_{1}, \lambda_{2} \in V(\tau)$, and $P$ a train path in $\tau$ with n-edges. Let $\mu_{1}, \mu_{2}$ be the MGL's constructed from $\left(\tau, \lambda_{1}\right),\left(\tau, \lambda_{2}\right)$ respectively, and let $\mathscr{P}_{1}, \mathscr{P}_{2}$ be the packets contained in $\mu_{1}$ and $\mu_{2}$ respectively, corresponding to $P$. Then:

$$
\left|\mu_{1}\left(\mathscr{P}_{1}\right)-\mu_{2}\left(\mathscr{P}_{2}\right)\right| \leq n\left|\lambda_{1}-\lambda_{2}\right|_{\infty}
$$

Proof.

$$
\left|\mu_{1}\left(\mathscr{P}_{1}\right)-\mu_{2}\left(\mathscr{P}_{2}\right)\right|=\left|\lambda_{1}(P)-\lambda_{2}(P)\right| \leq n\left|\lambda_{1}-\lambda_{2}\right|_{\infty}
$$

\section{Polynomial growth estimate}

Let $(\tau, \lambda)$ be a transversely recurrent train track on $R$ and $\mu$ its associated MGL. Let $R(n)$ denote the number of train paths in $\tau$ with $n$ edges that have geometric realizations as (non-empty) packets of leaves in $\mu$. A priori, $R(n)$ grows exponentially in $n$, since you can turn left or right at each switch.

Birman and Series [BS] have devised a clever coding scheme for simple geodesic arcs on a Riemann surface. Although their coding scheme was devised expressly to encode simple geodesic arcs on a Riemann surface, we use it to encode train paths (leaf packets) and prove that $R(n)$ grows as a polynomial in $n$. This distinction between subexponential/polynomial is essential to our later analysis.

THEOREM 1.3. Let $(\tau, \lambda)$ be a transversely recurrent train track on $R$ and $\mu$ its associated $M G L$. Let $R(n)$ denote the number of train paths in $\tau$ with $n$ edges that have geometric realizations as (non-empty) packets of leaves in $\mu$. Then:

$$
R(n) \leq C n^{(12 g-6)+2} \text {, and } C=C(g, \tau, \text { fixed fundamental polygon for } R) .
$$

Our strategy is to show a bounded correspondence between train track coding and Birman-Series coding of train paths. The proposition then readily follows since the Birman-Series coding has the polynomial growth built in. Most of the following is taken verbatim from [BS].

Choose and fix a convex fundamental polygon $\mathscr{D} \subset \mathbb{H}^{2}$ for $\pi_{1}(R)$.

In [BS], the authors show that the set of simple geodesics on $R$ is a nowhere dense subset of $R$ (and has measure 0 ). Using their result, we can choose $\mathscr{D}$ such that no simple geodesic intersects any vertex of $\mathscr{D}$ and $\partial \mathscr{D}$ does not contain any simple geodesic arcs. This simplification is not essential, but it gives cleaner statements of results.

Let $\mathrm{A}$ denote the set of sides of $\mathscr{D}$, ordered in any fixed manner.

Let $J_{\mu}$ denote the set of oriented simple geodesic arcs $\gamma \subset \mu$ such that $\partial \gamma \subseteq \pi(\partial \mathscr{D})$ and no subarc of $\gamma$ coincides with a side of $\pi(\partial \mathscr{D})$, where $\pi \in \mathbb{H}^{2} \rightarrow R$ is the universal covering map.

We refer to the components of $\gamma \cap \pi(\mathscr{D})$ as the segments of $\gamma$ and the points of $\gamma \cap \pi(\partial \mathscr{D})$ as the partition points of $\gamma$. We label the partition points $t_{0}, t_{1}, \ldots, t_{n}$ 
in the order in which they occur along $\gamma$ and we set $\|\gamma\|=n$. It is clear that any lift of $\gamma$ enters $n$ translates of $\mathscr{D}$.

Let $B=A \times A-\{$ diagonal $\}$ and define $h_{1}: J_{\mu} \rightarrow \mathbb{Z}^{B}, h_{1}(\gamma)=\left\{n\left(a, a^{\prime}\right)\right\}_{\left(a, a^{\prime}\right) \in B}$, where $n\left(a, a^{\prime}\right)$ is the number of segments joining $a$ to $a^{\prime}$ in (the lift of $\gamma$ to) $\mathscr{D}$. Clearly,

$$
\|\gamma\|=\sum_{\left(a, a^{\prime}\right) \in B} n\left(a, a^{\prime}\right) .
$$

When no confusion will occur, we will henceforth not distinguish between an arc $\gamma$ on $R$ and its lift to a broken arc on $\mathscr{D}$.

We shall need to record information about the position of the initial and final points $t_{0}, t_{n}$ of $\gamma$. For $i=0, n$ let $a\left(t_{i}\right)$ be the element of $A$ containing $t_{i}$ and let $j\left(t_{i}\right) \in N$ be the position of $t_{i}$ among the partition points of $\gamma$ which lie along $a\left(t_{i}\right)$ counting in the anticlockwise direction around $\partial \mathscr{D}$. Now define:

$$
\begin{gathered}
h_{2}: J_{\mu} \rightarrow(A \times Z)^{2} \\
h_{2}(\gamma)=\left(a\left(t_{0}\right), j\left(t_{0}\right), a\left(t_{n}\right), j\left(t_{n}\right)\right)
\end{gathered}
$$

and let

$$
h=\left(h_{1}, h_{2}\right): J_{\mu} \rightarrow Z^{B} \times(A \times Z)^{2}=\Omega .
$$

For

$$
w \in \Omega \text {, let }|w|=\sum_{\left(a, a^{\prime}\right) \in B}\left|w_{a, a^{\prime}}\right|,
$$

where $w_{a, a^{\prime}}$ is the projection of $w$ on the $\left(a, a^{\prime}\right)$ factor of $Z^{B}$. For $\gamma \in J_{\mu}$, define $\|\gamma\|_{B S}=|h(\gamma)|$. For $m \in N$, let $\Omega_{m}=\{w \in \Omega:|w| \leq m\}$.

LEMMA BS1. [BS, Lemma 2.1.] Suppose that $\gamma, \gamma^{\prime} \in J_{\mu}$ and that $h(\gamma)=h\left(\gamma^{\prime}\right)$. Let $t_{0}, \ldots, t_{n}$ and $t_{0}^{\prime}, \ldots, t_{n}^{\prime}$ be the partition points of $\gamma, \gamma^{\prime}$. Then $t_{i}$ and $t_{i}^{\prime}$ lie in the same element of $A$ for each $i=0,1, \ldots, n$, hence $\gamma$ and $\gamma^{\prime}$ are homotopic rel open edges in $\pi(\partial \mathscr{D})$.

LEMMA BS2. [BS, Lemma 2.2.]

$$
\#\left(\Omega_{n} \cap \text { Image }(h)\right) \leq C n{ }^{(\# A)+2},
$$

where $C$ depends on $\mathscr{D}$.

THEOREM. [BE]. Let $G$ be a finitely generated Fuchsian group of the first kind and let $P$ be any convex fundamental polygon for $G$. Suppose that $P$ has $N$ sides. Then $4 g \leq N \leq 12 g-6$.

Applying this theorem to Lemma BS2 yields:

$$
\left.\#\left(\Omega_{n} \cap \operatorname{Image}(h)\right) \leq C n^{(12 g-6}\right)+2 .
$$

Remark 1. The mapping $h:\left\{\gamma \subset J_{\mu}:\|\gamma\|_{\mathrm{BS}} \leq n\right\} \rightarrow\left(\Omega_{n} \cap\right.$ Image $\left.h\right)$ is a many-to-one mapping, however, by Lemma BS1, the mapping:

$$
h:\left\{\begin{array}{cc}
\text { Homotopy classes } & \|\gamma\|_{\mathrm{BS}} \leq n \text { and the homotopy } \\
\text { of } \gamma \subset J_{\mu} & \text { is rel open edges in } \pi(\partial \mathscr{D})
\end{array}\right\} \rightarrow\left(\Omega_{n} \cap \text { Image } h\right)
$$

is a one-to-one mapping. 
Remark 2. Using the coding scheme, we may formally code any simple arc $\gamma$ as long as $\partial \gamma \subset \pi(\partial \mathscr{D} \backslash$ vertices of $\mathscr{D}), \pi(\partial \mathscr{D})$ does not contain any subarc of $\gamma$, and $\gamma$ has no inessential intersections with $\mathscr{D}$. A moment's thought will show that Lemma BS 1 is valid if $\gamma$ and $\gamma^{\prime}$ are just simple arcs on $R$ and not necessarily geodesic arcs.

Remark 3. Let $P$ be a train path in $\tau$. Since $\partial P$ need not lie on $\pi(\partial \mathscr{D}), P$ may not have a unique coding, but could have as many as $(\# A)^{2}$ different codings corresponding to all possible extensions of the first and the last edge to $\pi(\partial \mathscr{D})$.

For the next proposition, we need a slightly more general notion of train path: we will drop the requirement that a train path must begin and end at a switch.

The following proposition shows that the correspondence between train paths in $\tau$ with geometric realizations and Birman-Series codes is at most $N$ to 1 .

LEMMA 1.4. Let $(\tau, \lambda)$ be a transversely recurrent train track such that $\tau$ does not intersect any vertex of $\pi(\mathscr{D}), \pi(\partial \mathscr{D})$ does not contain any subarc of $\tau$, and no train path in $\tau$ has an inessential intersection with $\mathscr{D}$, and let $\mu$ be its associated $M G L$. There exists $N=N(\mathscr{D}, \tau)$ such that if $P_{1}, \ldots, P_{r}$ are distinct train paths in $\tau$ which correspond to leaf packets in $\mu, \partial P_{k} \subset \pi(\partial \mathscr{D})$, and $h\left(P_{1}\right)=\cdots=h\left(P_{r}\right)$, then $r \leq N$.

Proof. Suppose $P_{1}, \ldots, P_{r}$ are distinct train paths in $\tau$ which correspond to leaf packets in $\mu, \partial P_{k} \subset \pi(\partial \mathscr{D})$, and $h\left(P_{1}\right)=\cdots=h\left(P_{r}\right)$. Studying fundamental constructions 1 and 2 carefully, one can show that $\mu$ can be collapsed onto $\tau$ in the following strong way: there exists a $C^{1}$ homotopy $F: I \times R \rightarrow R$ and $\varepsilon>0$ such that:

(a) $F(0, p)=p$ for all $p \in R$.

(b) $F(1, \mu) \subset \tau$.

(c) $F(*, \mu) \subset N_{\varepsilon}(\mu)$, where $N_{\varepsilon}(\mu)$ is the $\varepsilon$-neighborhood of $\mu$.

(d) $F(*$, leaf) is transverse to the foliation $\mathscr{F}$ obtained in Fundamental Construction \#1.

Choose geodesic arcs $\alpha_{1}, \ldots, \alpha_{r} \subset J_{\mu}$ which collapse onto $P_{1}, \ldots, P_{r}$ via the homotopy described above.

Unfortunately $h\left(\alpha_{k}\right)$ might not be equal to $h\left(P_{k}\right)$, since when we lift these curves to $\mathscr{D}$, 'glancing' may occur near a vertex. However, using property (d) of the homotopy described above, we can find simple $\operatorname{arcs} \beta_{1}, \ldots, \beta_{r}, \partial \beta_{k} \subset \pi(\partial \mathscr{D})$, such that $\beta_{k}$ collapses onto $P_{k}$ and $h\left(\beta_{k}\right)=h\left(P_{k}\right)$. By hypothesis $h\left(\beta_{1}\right)=\cdots=h\left(\beta_{r}\right)$. Lemma BS1 implies that $\beta_{1}, \ldots, \beta_{r}$ are all homotopic rel open edges of $\pi(\partial \mathscr{D})$, hence $P_{1}, \ldots, P_{r}$ are all homotopic rel open edges of $\pi(\partial \mathscr{D})$. If $P_{1}, \ldots, P_{r}$ were infinite train paths we could conclude that $P_{1}=\cdots=P_{r}[\mathbf{H P}]$. Since $P_{1}, \ldots, P_{r}$ are

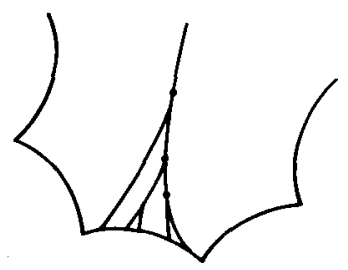

FIGURE 11. 'Last fundamental domain'. 
finite train paths, we can only conclude that (the lifts of) $P_{1}=\cdots=P_{r}$ outside of the first $l$ and last $m$ fundamental domains, where $l$ and $m$ are determined by $\mathscr{D}$ and $\tau$ and independent of the number of edges of the train paths. We choose $N$ to be the total number of train paths in the lift of $\tau$ contained in $\mathscr{D}$ with first and last edges of $\partial \mathscr{D}^{l+m}$.

Proof of Theorem 1.3. We will assume that $\tau$ does not intersect any vertex of $\pi(\mathscr{D})$, $\pi(\partial \mathscr{D})$ does not contain any subarc of $\tau$, and no train path in $\tau$ has an inessential intersection with $\pi(\partial \mathscr{D})$. This is no restriction, since we can always find a fundamental polygon for $R$ which satisfies the above conditions with respect to the fixed train track $\tau[\mathbf{N}]$.

$$
R(n) \leq \#\left\{\begin{array}{l}
P: P \text { is a train path in } \tau, P \text { corresponds to a leaf packet } \\
\text { in } \mu, \text { the first and last edges (or switches) intersect } \\
\pi(\partial \mathscr{D}), \text { and if } \bar{P} \text { is the retraction of } P \text { such that } \\
\partial(\bar{P}) \subset \pi(\mathscr{D}), \text { then }\|\bar{P}\|_{\mathrm{BS}} \leq n
\end{array}\right\}
$$

By Lemma 1.4,

$$
R(n) \leq N \#\left(\Omega_{n} \cap \text { Image }(h)\right),
$$

and by Lemma BS2,

$$
R(n) \leq N C n^{(12 g-6)+2}
$$

9. The geometry of fundamental construction $\# 2$

In the proof of the next theorem, we will need the following easy lemmas:

LEMMA 1.6. For large $b, \cos \theta \approx 2 \tanh (a / 2) e^{-b / 2}$.

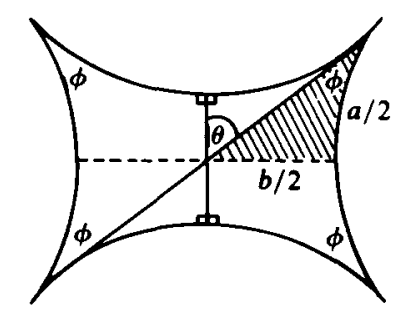

Figure 12

Proof. Since the shaded triangle is a right triangle, [BE]

$$
\begin{gathered}
\tan \left(\frac{\pi}{2}-\theta\right)=\frac{\tanh (a / 2)}{\sinh (b / 2)}=\cot \theta \\
\cos \theta=\frac{\cot \theta}{\sqrt{1+\cot ^{2} \theta}}=\frac{1}{\sqrt{\frac{\sinh ^{2}(b / 2)}{\tanh ^{2}(a / 2)}+1}} \stackrel{b \ll 1 \tanh (a / 2)}{\approx \sinh (b / 2)} \approx 2 \tanh (a / 2) e^{-b / 2}
\end{gathered}
$$

LEMMA 1.7. Let $(\tau, \lambda)$ be a transversely recurrent train track and let $\mu$ be its associated 
MGL. The hyperbolic length of a subleaf in $\mu$ is commensurable with the number of edges in $\tau$ it collapses onto, counted with multiplicity.

Proof. For each edge $e \subset \tau$, let

$$
\begin{aligned}
\mathscr{C}_{e}= & \{\alpha \text { subleaf of } \mu: \alpha \text { collapses onto } e \text { under the homotopy } \\
& \text { described in Lemma 1.4 }\} .
\end{aligned}
$$

Let $l^{*} \subset \mu$, and suppose $l^{*}$ collapses onto $n$ edges of $\tau$ (counted with multiplicity), then

$$
n\left[\min _{e \subset \tau} \inf _{\alpha \subset \mathscr{C}_{e}} l_{\mathrm{HYP}}(\alpha)\right] \leq l_{\mathrm{HYP}}\left(l^{*}\right) \leq n\left[\max _{e \subset \tau} \sup _{\alpha \in \mathscr{B}_{e}} l_{\mathrm{HYP}}(\alpha)\right]
$$

THEOREM 1.8. Let $\tau$ be a transversely recurrent train track on $R, \lambda_{1}, \lambda_{2} \in V(\tau), \mu_{1}$ and $\mu_{2}$ the associated MGL's, and t be any simple geodesic arc transverse to $\mu_{1}$ and $\mu_{2}$ which collapses onto one edge of $\tau$. Then:

$$
\left|\int_{1} \cos \theta d \mu_{1}-\int_{t} \cos \theta d \mu_{2}\right| \leq C\left|\lambda_{1}-\lambda_{2}\right|_{\infty} \log ^{r}\left(\frac{1}{\left|\lambda_{1}-\lambda_{2}\right|_{\infty}}\right),
$$

where $r=\left({ }^{12 g-6}\right)+3$, and $C$ depends on $\tau, t, \mu_{1}(t)$ and $\mu_{2}(t)$.

Proof. Let $c_{1}$ and $c_{2}$ be the collapsing maps for $\mu_{1}$ and $\mu_{2}$ onto $\tau$. We may assume that $c_{1}$ and $c_{2}$ collapse $t$ onto the same edge $e \subset \tau$. Let $P^{n}$ be a train path in $\tau$, consisting of $2 n+1$ edges, having $e$ as its middle edge, and let $\mathscr{P}_{k}^{n}$ denote the packets of leaves in $\mu_{k}$ which collapse onto $P^{n}$. Lift $\mu_{1}, \mu_{2}$ and $\tau$ to $H^{2}$ along with a fixed lift of $t$ which we denote $\tilde{t}^{*}$. Then:

$$
\begin{aligned}
& \left|\int_{1} \cos \theta d \mu_{1}-\int_{1} \cos \theta d \mu_{2}\right|=\left|\sum_{P^{n}}\left(\int_{i^{*}} \cos \theta d\left(\mu_{1} \cap \mathscr{P}_{1}^{n}\right)-\int_{i^{*}} \cos \theta d\left(\mu_{2} \cap \mathscr{P}_{2}^{n}\right)\right)\right| \\
& =\left|\sum_{\substack{P^{n}: \\
\mathscr{P}_{1}^{n} \neq \varnothing \text { and } \mathscr{P}_{2}^{n} \neq \varnothing}}\left(\int_{\tilde{i}^{*}} \cos \theta d\left(\mu_{1} \cap \mathscr{P}_{1}^{n}\right)-\int_{\tilde{i}^{*}} \cos \theta d\left(\mu_{2} \cap \mathscr{P}_{2}^{n}\right)\right)\right| \\
& +\left|\sum_{P^{n}:}\left(\int_{i^{*}} \cos \theta d\left(\mu_{1} \cap \mathscr{P}_{1}^{n}\right)-\int_{i^{*}} \cos \theta d\left(\mu_{2} \cap \mathscr{P}_{2}^{n}\right)\right)\right| . \\
& \begin{array}{c}
\mathscr{P}_{1}^{n} \neq \varnothing \text { and } \mathscr{S}_{2}^{n}=\varnothing \text { or } \\
\mathscr{P}_{1}^{n}=\varnothing \text { and } \mathscr{P}_{2}^{n} \neq \varnothing
\end{array}
\end{aligned}
$$

We will refer to those leaf packets which occur in the first sum as type 1 packets and those appearing in the second sum as type 2 packets. We being by estimating the first sum. By the triangle inequality, it follows that:

$$
\begin{aligned}
(1) \leq & \sum_{\substack{P^{n} \\
\text { type 1 }}} \int_{i^{*}}\left|\cos \theta-\left(\begin{array}{c}
\min \cos \\
\mathscr{P}_{1}^{n} \cap \tilde{t}^{*} \\
\mathscr{P}_{2}^{n} \cap \tilde{t}^{*}
\end{array}\right)\right| d\left(\mu_{1} \cap \mathscr{P}_{1}^{n}\right) \\
& +\sum_{P^{n}} \int_{i^{*}}\left|\cos \theta-\left(\begin{array}{c}
\min \cos \\
\mathscr{P}_{1}^{n} \cap \tilde{t}^{*} \\
\mathscr{P}_{2}^{n} \cap \tilde{t}^{*}
\end{array}\right)\right| d\left(\mu_{2} \cap \mathscr{P}_{2}^{n}\right) \\
& +\sum_{\substack{P^{n} \\
\text { type 1 }}}\left|\left(\begin{array}{l}
\min \cos \\
\mathscr{P}_{1}^{n} \cap \tilde{t}^{*} \\
\mathscr{P}_{2}^{n} \cap \tilde{t}^{*}
\end{array}\right)\right| \mid \int_{\tilde{i}^{*}} d\left(\mu_{1} \cap \mathscr{P}_{1}^{n}\right)-\int_{i^{*}} d\left(\mu_{2} \cap \mathscr{P}_{2}^{n} \mid .\right.
\end{aligned}
$$


Suppose $P^{n}=\left(e_{0}, \ldots, e_{n-1}, e, e_{n+1}, \ldots, e_{2 n+1}\right)$ is a type 1 train path. Let $v_{0}$ and $v_{2 n+1}$ be its initial and terminal switches. $\mathscr{P}_{k}^{n}$ connects $c_{k}^{-1}\left(v_{0}\right)$ to $\tilde{t}^{*}$ to $c_{k}^{-1}\left(v_{2 n+1}\right)$. Since the collection $\left\{c_{k}^{-1}(v)\right\}_{k=1,2}$, where $v$ is a switch in $\tau$, is finite, Lemmas 1.6 and 1.7 imply there exists constants $c_{1}$ and $c_{2}$ such that:

Hence:

$$
\left|\left(\begin{array}{c}
\max \cos \\
\mathscr{P}_{1}^{n} \cap \tilde{t}^{*} \\
\mathscr{P}_{2}^{n} \cap \tilde{t}^{*}
\end{array}\right)-\left(\begin{array}{c}
\min \cos \\
\mathscr{P}_{1}^{n} \cap \tilde{t}^{*} \\
\mathscr{P}_{2}^{n} \cap \tilde{t}^{*}
\end{array}\right)\right| \leq c_{1} e^{-c_{2} n}
$$

$$
\begin{aligned}
(1) \leq & \sum_{\substack{P^{n} \\
\text { type } 1}} c_{1} e^{-c_{2} n} \int_{\tilde{i}^{*}} d\left(\mu_{1} \cap \mathscr{P}_{1}^{n}\right)+\sum_{\substack{P^{n} \\
\text { type } 1}} c_{1} e^{-c_{2} n} \int_{\tilde{i}^{*}} d\left(\mu_{2} \cap \mathscr{P}_{2}^{n}\right) \\
& +\sum_{\substack{P^{n} \\
\text { type } 1}}\left|\int_{\tilde{i}^{*}} d\left(\mu_{1} \cap \mathscr{P}_{1}^{n}\right)-\int_{\tilde{i}^{*}} d\left(\mu_{2} \cap \mathscr{P}_{2}^{n}\right)\right| \\
\leq & c_{1} e^{-c_{2} n} \mu_{1}\left(\tilde{t}^{*}\right)+c_{1} e^{-c_{2} n} \mu_{2}\left(\tilde{t}^{*}\right)+\underset{\underset{P^{n}}{\text { type 1 }}}{ }\left|\int_{\tilde{i}^{*}} d\left(\mu_{1} \cap \mathscr{P}_{1}^{n}\right)-\int_{\tilde{i}^{*}} d\left(\mu_{2} \cap \mathscr{P}_{2}^{n}\right)\right|
\end{aligned}
$$

The packet uncertainty principle (Corollary 1.2 ) implies that:

$$
\left|\int_{i^{*}} d\left(\mu_{1} \cap \mathscr{P}_{1}^{n}\right)=\int_{i^{*}} d\left(\mu_{2} \cap \mathscr{P}_{2}^{n}\right)\right| \leq(2 n+1)\left|\lambda_{1}-\lambda_{2}\right|_{\infty} .
$$

The number of type 1 packets is bounded by the total number of packets, $R(2 n+1)$, which by Theorem 1.3 is bounded by $c_{3} n^{r}$, where $r=\left(\frac{12 g-6}{2}\right)+2$. Thus, we may estimate the first sum by:

$$
\text { (1) } \leq c_{1} e^{-c_{2} n}\left(\mu_{1}\left(\tilde{t}^{*}\right)+\mu_{2}\left(\tilde{t}^{*}\right)\right)+(2 n+1)\left|\lambda_{1}-\lambda_{2}\right|_{\infty} c_{3} n^{r} .
$$

We will assume that $\mu_{1}\left(\tilde{t}^{*}\right)$ and $\mu_{2}\left(\tilde{t}^{*}\right)$ are bounded by some constant, hence,

$$
(1) \leq c_{4} e^{-c_{2} n}+(2 n+1)\left|\lambda_{1}-\lambda_{2}\right|_{\infty} c_{3} n^{r} \text {. }
$$

The second sum may be crudely estimated by:

$$
(2) \leq \sum_{\substack{\boldsymbol{P}^{n} \\ \text { type 2 }}} \int_{i^{*}} d\left(\mu_{l} \cap \mathscr{P}_{l}^{n}\right)
$$

where the choice of $l$ depends on the particular packet, $l=1$ or 2 .

It is an immediate consequence of the packet uncertainty principle (Corollary 1.2) that for type 2 packets,

$$
\int_{i^{*}} d\left(\mu_{l} \cap \mathscr{P}_{l}^{n}\right) \leq(2 n+1)\left|\lambda_{1}-\lambda_{2}\right|_{\infty}
$$

Unfortunately, we have no control over the number of type 2 packets. Again, all we know is the number of type 2 packets is bounded by the total number of packets $R(2 n+1)$, which by Theorem 1.3 is bounded by $c_{3} n^{r}$, where $r=\left(\begin{array}{c}12 g-6 \\ 2\end{array}\right)+2$. Thus, we may estimate the second sum by:

$$
(2) \leq(2 n+1)\left|\lambda_{1}-\lambda_{2}\right|_{\infty} c_{3} n^{r}
$$

It follows that the entire sum may be estimated by:

$$
(1)+(2) \leq c_{4} e^{-c_{2} n}+2(2 n+1)\left|\lambda_{1}-\lambda_{2}\right|_{\infty} c_{3} n^{r} .
$$


Equating the two terms, we choose

$$
n=\frac{1}{c_{2}} \log \left(\frac{c_{4}}{4 c_{3}} \frac{1}{\left|\lambda_{1}-\lambda_{2}\right|_{\infty}}\right),
$$

and after absorbing constants, we obtain:

$$
\begin{aligned}
(1)+(2) \leq & c_{5}\left|\lambda_{1}-\lambda_{2}\right|_{\infty}+c_{6}\left|\lambda_{1}-\lambda_{2}\right|_{\infty} \log ^{r+1}\left(c_{7} \frac{1}{\left|\lambda_{1}-\lambda_{2}\right|_{\infty}}\right) \\
& +c_{8}\left|\lambda_{1}-\lambda_{2}\right|_{\infty} \log ^{r}\left(c_{7} \frac{1}{\left|\lambda_{1}-\lambda_{2}\right|_{\infty}}\right)
\end{aligned}
$$

For $\left|\lambda_{1}-\lambda_{2}\right|_{\infty} \ll 1$,

$$
(1)+(2) \leq c_{9}\left|\lambda_{1}-\lambda_{2}\right|_{\infty} \log ^{r+1}\left(\frac{1}{\left|\lambda_{1}-\lambda_{2}\right|_{\infty}}\right) .
$$

Remark. This theorem may be interpreted as saying that if two MGL's have intersection numbers which are $\varepsilon$ close with respect to suitably chosen transverse arcs, then their total cosines with respect to closed geodesics are $C \varepsilon \log ^{r+1}(1 / \varepsilon)$ close.

\section{Earthquakes}

Thurston has generalized the Dehn twist or Fenchel-Nielsen deformation about a simple closed geodesic to a 'twisting' or 'shearing' about a MGL, called an earthquake deformation.

Recall that for $R$, the set $\mathscr{S} \times \mathbb{R}^{+}$consisting of weighted simple closed geodesics in $R$ is dense in $M L$. The Earthquake deformation about a MGL $\mu$ is defined by the limit in $\mathscr{T}_{g}$ of any sequence of Dehn twists along weighted simple closed geodesics which converge to $\mu$ in $M L$. Formally:

Definition. (Thurston.) The left Earthquake deformation of $R$ at time $t$ determined by $\mu \in M L$ is the limit in $\mathscr{T}_{\mathrm{g}}$ of the time $t$ (Dehn) twist deformations of $R$ for any sequence $\left(\alpha_{i}, \gamma_{i}\right) \in \mathscr{S} \times \mathbb{R}^{+}$converging to $\mu$ in $M L$. It will be denoted $E(R, \mu, t)$.

In [ $K_{1}$ ], Kerckhoff shows that this definition is valid, i.e., that the limits exist and are independent of the approximating sequence, and hence unique.

We will think of earthquake deformations as defining paths in $\mathscr{T}_{g}$ as $t$ varies.

THEOREM. (Kerckhoff, $\left[\mathbf{K}_{3}\right]$.) Every tangent vector in $\mathscr{T}_{g}$ is tangent to a unique earthquake path in $\mathscr{T}_{\mathbf{g}}$. In fact:

$$
\begin{aligned}
\psi: M L & \mapsto\left(\mathscr{T}_{\mathrm{g}}\right)_{R} \\
\mu & \left.\mapsto \frac{d}{d t}\right|_{\ell=0^{+}} E(R, \mu, t)
\end{aligned}
$$

is a homeomorphism.

Choose $6 g-6$ simple closed geodesics $\alpha_{1}, \ldots, \alpha_{6 g-6}$ whose lengths $l_{\alpha_{1}}, \ldots, l_{\alpha_{6 g-6}}$ serve as local coordinates in $R \in U \subset \mathscr{T}_{\mathrm{g}}$. In these coordinates,

$$
\left.\frac{d}{d t}\right|_{t=0^{+}} E(R, \mu, t)=\sum_{k=1}^{6 g-6}\left[\left.\frac{d}{d t}\right|_{t=0^{+}} l_{\alpha_{k}}(E(R, \mu, t))\right] \frac{\partial}{\partial l_{\alpha_{k}}} .
$$


In $\left[\mathbf{K}_{\mathbf{2}}\right]$, Kerckhoff shows:

$$
\left.\frac{d}{d t}\right|_{t=0^{+}} l_{\alpha_{k}}(E(R, \mu, t))=\cos \left(\alpha_{k}, \mu\right)=\int_{\alpha_{k}} \cos \theta d \mu
$$

hence,

$$
\left.\frac{d}{d t}\right|_{t=0^{+}} E(R, \mu, t)=\sum_{k=1}^{6 g-6}\left(\int_{\alpha_{k}} \cos \theta d \mu\right) \frac{\partial}{\partial l_{\alpha_{k}}} .
$$

Definition. $f: \mathbb{R}^{m} \rightarrow \mathbb{R}^{n}$ is ' $k$ Log Hölder' iff there exists $C, K$ such that

$$
|f(x)-f(y)| \leq C|x-y| \log ^{K}\left(\frac{1}{|x-y|}\right) .
$$

Remark. It is easy to show that $k$ Log Hölder implies $\alpha$-Hölder continuous for all $\alpha<1$, but it does not imply Lipshitz.

Theorem 1.8 applied to $\alpha_{1}, \alpha_{2}, \ldots, \alpha_{6 g-6}$ readily implies:

Corollary 1.9. $\psi: M L(R) \rightarrow\left(\mathscr{T}_{\mathrm{g}}\right)_{R}$ is $k$ Log Hölder.

\section{Acknowledgments}

Most of the contents of this paper appears in the first half of my thesis. I would like to thank my advisor, Scott Wolpert, for his expert guidance and encouragement, and the Alfred P. Sloan Foundation for supporting this work through a Doctoral Dissertation Fellowship. I would also like to thank the referee for making several valuable suggestions.

\section{REFERENCES}

[BE] A. Beardon. The Geometry of Discrete Groups Springer Verlag: New York, NY, 1983.

[BS] J. Birman \& C. Series. Simple curves have Hausdorff dimension one. Preprint.

[HP] J. Harer \& R. Penner. Combinatorics of train tracks. To appear.

[K ] S. Kerckhoff. The Nielsen realization problem. Ann. Math. 117 (1983), 235-265.

[K, S. Kerckhoff. Earthquakes are analytic. Comm. Math. Helvetici 60 (1985).

[ $\mathbf{K}_{3}$ ] S. Kerckhoff. Lines of Minima in Teichmüller space. To appear.

[MOO] R. L. Moore. Concerning upper semi-continuous collections of continua. Trans. Amer. Math. Soc. 27 (1925), 416-428.

[MOR] J. Morgan. Columbia University Lecture Notes.

[N] P. Nicholls. Transitivity properties of fuchsian groups. Canad. J. Math. XXVIII \#4 (1976), 805-814.

[T] W. P. Thurston. The geometry and topology of 3-manifolds. Princeton University Lecture Notes.

[ $\left.\mathbf{W}_{1}\right]$ S. Wolpert. University of Maryland Lecture Notes. 